\title{
Satisfaire les besoins de santé des hommes qui ont des rapports sexuels avec d'Autres hommes au Sénégal
}

Horizons Program

Follow this and additional works at: https://knowledgecommons.popcouncil.org/departments_sbsr-hiv

Part of the Gender and Sexuality Commons, and the International Public Health Commons How does access to this work benefit you? Let us know!

\section{Recommended Citation}

"Satisfaire les besoins de santé des hommes qui ont des rapports sexuels avec d'Autres hommes au Sénégal," Horizons Resume de Recherche. Dakar: Population Council, 2002. 


\section{Satisfaire les Besoins de Santé des Hommes Qui ONT Des Rapports Sexuels Avec D'Autres Hommes au Sénégal}
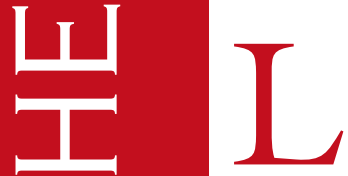

a recherche effectuée dans de nombreux pays a souligné la vulnérabilité des hommes qui ont des rapports sexuels avec d'autres hommes (MSM) face au VIH et à d'autres infections sexuellement transmissibles (IST). Pourtant, en Afrique, peu d'attention est accordée à ces derniers dans les programmes et prestations contre le VIH/SIDA, à cause du désaveu et de la stigmatisation qui entourent communément le comportement homosexuel. Au Sénégal, une étude à ce sujet a été menée par le Programme National de Lutte contre le SIDA (PNLS), l'Université Cheikh Anta Diop, et le Programme Horizons. Celle-ci a revelé des informations sur les besoins, comportements, connaissances, et attitudes des MSM fort utiles aux decideurs et aux responsables de programmes oeuvrant pour le contrôle de la propagation du VIH/SIDA.

\section{Méthodes de Recherche}

Les chercheurs se sont servis de méthodes ethnographiques et d'enquêtes pour obtenir des informations des MSM âgés de plus de 18 ans dans plusieurs quartiers de Dakar. Dans la phase ethnographique, les chercheurs ont tenu des discussions de groupe et des interviews avec des MSM et des gens en interaction avec ces derniers, tels que les tenanciers de bars, les travailleuses du sexe, et les chauffeurs de taxi. Ils ont aussi observé différents sites, notamment des endroits où les MSM se rencontrent, et des rassemblements où ceux-ci jouent un rôle social reconnu comme les groupements féminins. Ils ont par ailleurs développé des études de cas de huit MSM.

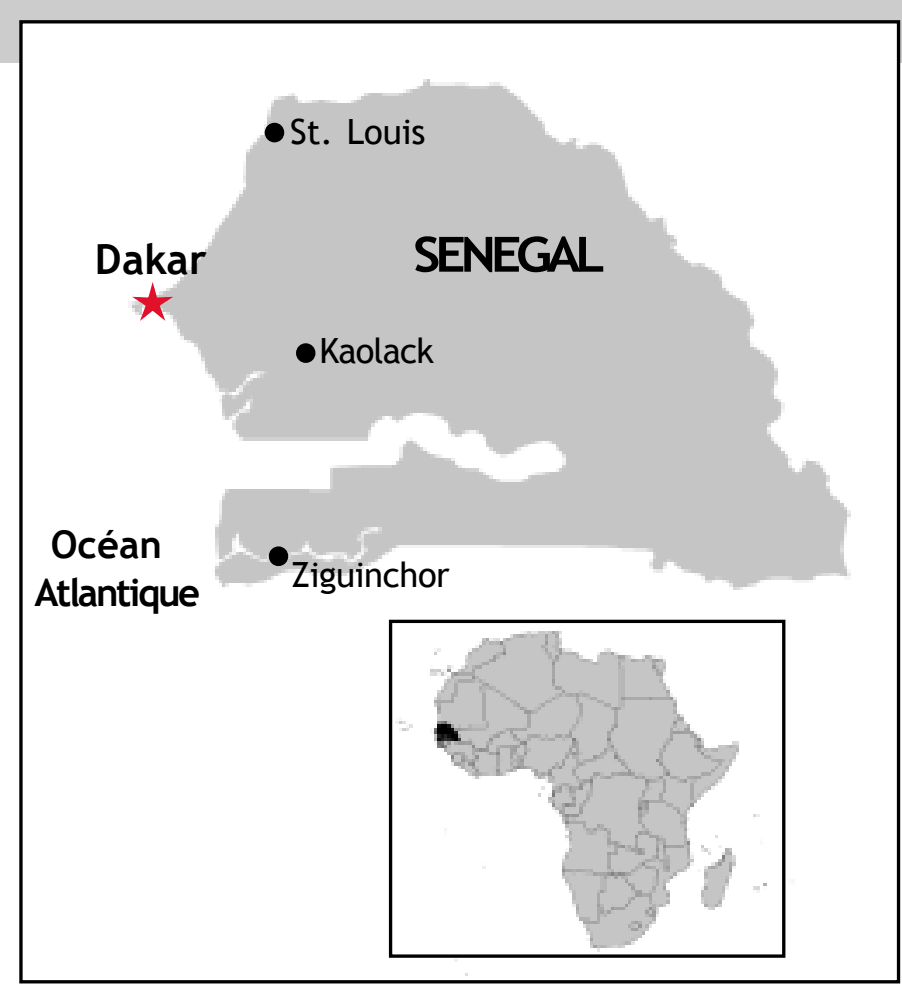

Dans la seconde phase, les chercheurs ont enquêté sur un échantillon de commodité de 250 MSM. La classe d'âge des enquêtés se situait entre 18 et 53 ans, avec un âge moyen de 25 ans. Quatre vingt deux pour cent $(82 \%)$ sont des célibataires et $15 \%$ ont déclaré être mariés ; certains étant dans des ménages polygames. Environ le quart $(1 / 4)$ des hommes avaient des enfants. Plus du tiers $(1 / 3)$ de l'échantillon appartient à l'ethnie Wolof, avec de nombreux autres groupes ethniques représentés. Le niveau d'éducation était relativement bas : 15\% n'avaient jamais été à l'école et $55 \%$ n'avaient pas terminé le cours primaire ; les enquêtés ont indiqué un éventail de niveaux de revenus (bas, moyen, et élevé) et d'occupations, notamment des sportifs professionnels, mécaniciens, artistes, journaliers, marabouts, et étudiants. Vingt quatre pour cent (24\%) étaient en chômage. 
L'équipe de recherche a recruté des informateurs en se rendant dans des lieux fréquentés par des MSM, en prenant contact et en développant une relation de confiance avec eux et certains parmi les leaders. Le processus de la recherche a conduit l'équipe de chercheurs à consacrer beaucoup de temps à l'analyse de leurs propres idées préconçues sur la sexualité, en opérant à travers les préjugés et tabous tout en soulignant l'importance du maintien de la confidentialité des informateurs. Toutes les interviews étaient anonymes et les informateurs ont accepté de donner leur accord verbalement en connaissance de cause. Une fois l'accord obtenu, les informateurs ont reçu une petite somme forfaitaire de 2500 FCFA pour couvrir leurs frais de transport, l'information sur les IST et le VIH/ SIDA et une référence pour une consultation médicale et un traitement gratuits.

\section{Principaux Résultats}

\section{Les MSM ont des identités et rôles sociaux} distincts qui dépassent le cadre des pratiques sexuelles. Défini largement, les Ibbis sont plus enclins à adopter des maniérismes féminins et à être moins dominateurs dans les interactions sexuelles. Bien que la société rejette formellement l'homosexualité, les Ibbis n'en bénéficient pas moins d'une grande considération dans certains milieux. Par exemple, les Ibbis peuvent souvent entretenir des relations avec des femmes dotées de pouvoir politique ou économique et pour lesquelles ils accomplissent d'importantes cérémonies et fonctions sociales. Dans plusieurs quartiers, les Ibbis jouissent de la protection de la communauté tout entière. Les Yoos sont généralement les partenaires pénétrant, mais ne se considèrent pas eux-mêmes comme des homosexuels. Au-delà de ces larges catégories, il existe d'autres sous-catégories basées sur l'âge, le statut, et le type de relation. Toutefois, l'identification à un groupe particulier ne constitue pas une indication infaillible des pratiques sexuelles d'un individu.
Tableau N¹: Pourcentage de MSM ayant subi des agressions ( $n=250)$

\begin{tabular}{|c|c|c|c|}
\hline & \multicolumn{3}{|c|}{ Source / Cadre de l'agression } \\
\hline & Famille & Communauté & Poste de Police \\
\hline $\begin{array}{l}\text { Agression } \\
\text { verbale }\end{array}$ & 49 & 40 & 19 \\
\hline $\begin{array}{l}\text { Agression } \\
\text { physique }\end{array}$ & 28 & 12 & 13 \\
\hline
\end{tabular}

La première expérience sexuelle se passe souvent avec un adulte, pendant l'adolescence. La moyenne d'âge à la première expérience sexuelle est de 15 ans. Cette expérience était souvent faite avec un adulte, quelqu'un dans le cercle des connaissances ou qui avait été récemment rencontré. Un tiers $(1 / 3)$ de l'échantillon a rapporté que l'adulte était un membre de la famille étendue de l'enquêté.

\section{La vie de beaucoup de MSM est caractérisée} par la violence et le rejet. Quarante trois pour cent $(43 \%)$ des MSM ont été violés au moins une fois en dehors de leur foyer familial ; $37 \%$ au cours des 12 derniers mois. Treize pour cent (13\%) révèlent avoir été violés par un policier. Près de la moitié des 250 hommes interrogés avait subi des agressions verbales (notamment des injures et des menaces) de la part de leur famille (Tableau 1 ). Beaucoup ont aussi fait état d'agressions physiques (exemples : coups, jets de pierre) de la part de leurs familles, de membres de la communauté, et de la police. L'étude a révélé une très grande mobilité parmi les hommes, aussi bien volontaire qu'involontaire; environ un quart (1/4) révélant avoir été contraint de déménager au cours des 12 derniers mois. Nombre d'entre eux ont souligné l'importance de maintenir le secret de leurs penchants et relations sexuelles parce qu'une éventuelle révélation de celles-ci les conduirait à l'ostracisme, la stigmatisation, les injures, ou les agressions physiques. Selon un informateur :

«Dans certains quartiers, lorsqu'on découvre que vous êtes un Ibbi, tandis que vous passez votre chemin, les gens se mettent en groupe pour vous jeter des pierres. Vous avez l'impression que c'est alors une pluie de pierres. » 
Les rapports sexuels avec les hommes sont dictés par plusieurs raisons dont l'amour, le plaisir et la contrepartie économique. Bon nombre de leurs histoires personnelles soulignent que la première expérience sexuelle tout comme les suivantes surviennent dans un contexte d'attirance émotionnelle et physique. Selon un MSM :

"Il m'a présenté à ses amis. Il est venu vers moi et m'a beaucoup caressé. J'ai ressenti énormément de plaisir. Je me sentais heureux et j'ai fini par avoir une relation d'amour très forte avec lui. »

La contrepartie économique joue aussi un rôle important. Les deux tiers $(2 / 3)$ des enquêtés rapportent avoir reçu de l'argent dans le cadre de leur plus récente rencontre sexuelle, et $9 \%$ avaient donné de l'argent en contrepartie de rapports sexuels. Un MSM a rapporté son expérience :

"Il m'a invité chez lui le jour suivant. Il m'a donné de l'argent... Au moment de nous quitter, il m'a encore donné beaucoup d'argent, vraiment beaucoup. Et il m'a demandé de revenir aussi souvent que je voulais, ce que j'ai accepté. Et j'ai pris goût au plaisir et à l'argent. Il s'occupait de toutes mes dettes. »

Les MSM ont fait état d'une vaste gamme de relations sexuelles allant de la relation stable et régulière avec un seul partenaire aux relations irrégulières avec plusieurs partenaires, et sans exclure la relation régulière avec un partenaire, en plus des partenaires occasionnels.

\section{La grande majorité des MSM ont eu des} rapports sexuels avec des femmes. Quatre vingt huit pour cent $(88 \%)$ de l'échantillon de l'enquête ont déclaré avoir déjà eu des rapports vaginaux, et prés du cinquième $(1 / 5)$ avaient pratiqué une pénétration anale avec une femme. Certaines de ces relations sexuelles ont eu lieu en contrepartie d'une somme d'argent versée : $21 \%$ des MSM ont déclaré avoir donné de l'argent lors du dernier rapport sexuel avec une femme et $13 \%$ d'entre ont déclaré en avoir reçu.

\section{Bon nombre de MSM sont à haut risque du VIH à cause des rapports sexuels non protégés, des antécédents de symptômes d'IST, et une mauvaise connaissance de ces infections. Lorsqu'ils ont été interrogés sur}

l'utilisation du condom lors de leur dernier rapport, seulement $23 \%$ de ceux pratiquant des rapports anaux ont déclaré avoir fait usage du condom. En revanche, la proportion est moindre chez les pénétrés : $14 \%$ d'entre eux ont fait usage du préservatif. L'utilisation du condom avec les femmes était également faible ; 37\% ont indiqué avoir fait usage du condom lors de leur dernier rapport avec une femme. Les informateurs ont identifié un certain nombre d'obstacles à l'utilisation du condom, notamment la diminution du plaisir, l'interférence avec l'établissement de la confiance, et un manque de pouvoir chez certains MSM, pour demander des préservatifs. De l'avis d'un informateur, «Si un Yoos ne veut pas utiliser un préservatif, un Ibbi ne peut pas dire grand chose. » Les informateurs ont également signalé le coût élevé des marques préférées de condoms et l'accès médiocre aux lubrifiants aqueux.

Presque tous les 250 MSM interrogés reconnaissaient que le VIH/SIDA peut être contracté à travers une relation sexuelle et $80 \%$ d'entre eux ont cité le condom comme un moyen de prévention, même si l'utilisation actuelle ne reflète pas cette connaissance.

Beaucoup d'enquêtés ont dit avoir connu des symptômes liés aux IST. Par exemple, $42 \%$ ont rapporté ressentir des brûlures ou écoulements péniens au moment de l'enquête et $22 \%$ ont révélé avoir des lésions ou pustules à l'anus (Tableau 2). En les interrogeant sur l'origine de ces infections, la plupart des enquêtés ont évoqué des causes non virales ou bactériennes telles que la mauvaise hygiène, l'irritation due au coït sans une lubrification suffisante, les aliments épicés, de longues périodes d'abstinence, la masturbation, trop de rapports sexuels, ou d'autres maladies. Plus du tiers a déclaré n'avoir aucune idée sur la cause.

Les comportements visant à rechercher des soins pour les symptômes d'IST sont souvent retardés et cachés. Les MSM ont noté qu'ils sont particulièrement réticents à l'idée de révéler des symptômes anaux dans les cliniques et hôpitaux parce qu'ils risquent de mettre à nu leur homosexualité. Certains d'entre eux ont noté que le personnel des centres médicaux les traitait avec mépris, s'il ne les ignorait pas tout simplement, et qu'il ne respectait aucunement leur confidentialité. Cela étant, certains informateurs affirment ne rien faire pour soigner leurs 
Tableau N2 : Expériences de symptômes d'IST $(\mathrm{n}=\mathbf{2 5 0})$

\begin{tabular}{|lc|}
\hline Symptôme & Pourcentage \\
\hline Ecoulement et brûlure au pénis & 42 \\
Saignement et écoulement à l'anus & 42 \\
Ganglions enflés à l'aine & 36 \\
Démangeaison et plaies autour du pénis & 24 \\
Plaies et boutons autour de l'anus & 22 \\
Testicules douloureux et enflés & 12 \\
\hline
\end{tabular}

symptômes, et font état d'automédication par le biais de remèdes achetés sans ordonnance.

Cependant, étant donné qu'il y a moins de stigmate associé aux symptômes péniens, tels que les écoulements, les brûlures, les démangeaisons et plaies, les MSM sont plus enclins à se rendre dans un hôpital ou clinique public pour le traitement de ces cas. A la question de savoir où ils préféreraient aller se faire soigner pour des symptômes anaux ou péniens, la majorité a répondu que leur préférence était les hôpitaux ou dispensaires publics, sous réserve de leur accessibilité financière et que les clients soient traités en toute confidentialité et respect.

\section{Conclusion et Prochaines Etapes}

Cette étude projette d'importants regards sur la sexualité des MSM, leur vulnérabilité au VIH/ IST, et le rôle de la violence et du stigmate dans leurs vies. Les résultats mettent aussi en évidence le manque de services de santé sexuelle et d'information disponible pour couvrir les besoins spécifiques des MSM.

Les résultats issus de cette étude ont fait l'objet d'une restitution au cours d'une réunion tenue en avril 2001 à Dakar, et ont servi de catalyseur à la prise de conscience sur l'importance pour la santé publique de développer des interventions non stigmatisantes pour les MSM. Il en résulte la mise sur pied d'un pouls d'ONGs chargé de développer et de coordonner les activités à l'intention des MSM à Dakar. Cette initiative a été possible grâce à la mission USAID sous les auspices du PNLS. Les éléments potentiels d'intervention sont notamment la communication pour provoquer des changements de comportement, le développement des capacités des leaders MSM, la formation de pairs éducateurs, l'identification de prestataires de services sensibilisés aux besoins de santé des MSM, et la création d'espaces qui offrent sécurité et confort et où les MSM peuvent se retrouver pour des échanges d'informations.

Novembre 2001.

Les chercheurs dans cette étude sont Cheikh Ibrahima Niang, Moustapha Diagne, Youssoupha Niang, Amadou Mody Moreau, Dominique Gomis, et Mayé Diouf de l'Institut des Sciences de l'Environnement de l'Université Cheikh Anta Diop ; Karim Seck et Abdoulaye Sidibé Wade du Programme National de Lutte contre le SIDA; et Placide Tapsoba de Horizons/Population Council.

Les chercheurs tiennent à remercier les institutions suivantes pour leur appui dans cette étude : Africa Consultant International (ACI), Organisation Panafricaine de Lutte contre le SIDA / Centre de Traitement Ambulatoire (OPALS/CTA), et FHI/Impact.

Pour de plus amples informations sur l'étude, contacter le Dr Niang (ciniang@sentoo.sn) ou le Dr Tapsoba (ptapsoba@pcaccra.org).

Vous pouvez obtenir une copie du prochain rapport complet en contactant Horizons Publications (horizons@pcdc.org).

\section{Hqrizons}

Population Council/Horizons

Communications Unit

4301 Connecticut Avenue, NW

Suite 280

Washington, DC 20008
Population Council

Tel: 202-237-9400

Fax: 202-237-8410 horizons@pcdc.org www.popcouncil.org/horizons
USAID xas

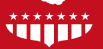
Cette publication a été rendue possible grâce à l'appui du Global Bureau de Santé / VIH-SIDA de l'Agence des Etats Unis pour le développement International (USAID) au terme du contrat $\mathrm{N}^{\circ} \mathrm{HRN}-\mathrm{A}-00-97-00012-00$. Les opinions exprimées ici incombent aux auteurs, et ne reflètent pas forcément les vues de l'USAID.

๑ 2002. The Population Council Inc. 